Published in "Brain and Language 119(3): 238-242, 2011"

which should be cited to refer to this work.

\title{
Language and syntactic impairment following stroke in late bilingual aphasics
}

\author{
Muriel Tschirren ${ }^{\mathrm{a}}$, Marina Laganaro ${ }^{\mathrm{c}}$, Patrik Michel ${ }^{\mathrm{d}}$, Marie-Dominique Martory ${ }^{\mathrm{e}}$, Marie Di Pietro ${ }^{\mathrm{a}}$, \\ Jubin Abutalebi ${ }^{\mathrm{d}}$, Jean-Marie Annoni ${ }^{\mathrm{a}, \mathrm{b}, \mathrm{e}, *}$
}

a Department of Clinical Neurosciences, Geneva University Hospitals and Faculty of Medicine, Switzerland

${ }^{\mathrm{b}}$ Department of Neurosciences, Centre Hospitalier Universitaire Vaudois, Lausanne, Switzerland

${ }^{\mathrm{c}}$ Faculty of Psychology, University of Geneva, Switzerland

d Psychology Faculty, University Vita-Salute San Raffaele and Scientific Institute San Raffaele, Milano, Italy

${ }^{\mathrm{e}}$ Neurology Unit, Department of Medicine, University of Fribourg, Fribourg, Switzerland

\begin{abstract}
Purpose: Bilingual aphasia generally affects both languages. However, the age of acquisition of the second language (L2) seems to play a role in the anatomo-functional correlation of the syntactical/grammatical processes, thus potentially influencing the L2 syntactic impairment following a stroke. The present study aims to analyze the influence of late age of acquisition of the L2 on syntactic impairment in bilingual aphasic patients.

Methods: Twelve late bilingual participants (speaking French as L2 and either English, German, Italian or Spanish as L1) with stroke-induced aphasia participated in the study. The MAST or BAT aphasia batteries were used to evaluate overall aphasia score. An auditory syntactic judgement task was developed and used to test participants syntactic performance.

Results: The overall aphasia scores did not differ between L1 and L2. In a multiple case analysis, only one patient had lower scores in L2. However, four patients presented significantly lower performances in syntactic processing in the late L2 than in their native language (L1). In these four patients the infarct was localized, either exclusively or at least partially, in the pre-rolandic region.

Conclusion: This pilot study suggests that, in late bilingual aphasics, syntactic judgment abilities may be more severely impaired in L2, and that this syntactic deficit is most likely to occur following anterior lesions.
\end{abstract}

\section{Introduction}

Bilingualism is often defined as the regular use of two languages in everyday life (Grosjean, 1992). The overlapping of cortical representation between the native (L1) and the second languages (L2) in bilingual speakers is widely accepted (Abutalebi, 2008; Perani \& Abutalebi, 2005). The degree of this overlap appears to be influenced by several biographical factors, such as the age of acquisition (AoA), the degree of language proficiency and exposure (see for reviews: Abutalebi \& Green, 2007; Indefrey, 2006; Perani \& Abutalebi, 2005).

Biographical factors are also known to affect lexical-semantic and syntactic processes or representations (Weber-Fox \& Neville, 1996). While lexical processing is proficiency-dependent, syntactic processing is thought to depend mostly upon the age of L2 acquisition (Wartenburger et al., 2003; Weber-Fox \& Neville, 1996). One hypothesis is that grammatical processing of a late learned L2

\footnotetext{
* Corresponding author at: Neurology Unit, Department of Medicine, University of Fribourg, Fribourg, Switzerland.

E-mail address: jean-marie.annoni@unifr.ch (J.-M. Annoni).
}

relies on explicit processing demands, since learning acquisition involves declarative memory resources. By contrast, syntactic processing of L1 is based on implicit processing (Ullman, 2001). Implicit processing is also specific to L2 in "early bilinguals" i.e., bilinguals who learned their L2 before the so-called "critical period" between 5 and 7 years old (Singleton, 2007). A difference between implicit and explicit syntactic/grammatical learning was also postulated at the neural level: implicit memory is generally subserved by fronto-subcortical loops implicating left frontal and basal ganglia structures, whereas explicit processing is associated with bilateral temporal (particularly lateral) lobes structures (Ullman, 2001). Initial evidence for a difference in syntactic processing in L1 and L2 comes from the fact that bilingual Parkinson's Disease patients show greater syntactic impairments in L1 than L2 (if formally learned), suggesting a major involvement of procedural memory in representing L1 grammar (Zanini et al., 2004).

Aphasia studies in bilingual speakers show different patterns of impairment and recovery for the two languages (Ansaldo, Marcotte, Scherer, \& Raboyeau, 2008; Weekes, 2010). Most patients display a parallel total recovery rate (65\%) (Fabbro, 2001; 
Green, 1995; Paradis, 2001), at least in early bilinguals. However, to the best of our knowledge, specific studies of late bilinguals which evaluate language modalities and components separately (i.e., lexico-semantic, syntax, phonology) are still limited.

This present pilot study was designed to investigate the influence of AoA upon L2 syntactic processing in bilingual aphasics. Specifically, our main purpose was to investigate whether anterior brain lesions may leave L2 syntactic processing relatively unaffected, as suggested by Ullman (2001).

\section{Results}

\subsection{Aphasia scores in $L 1$ and $L 2$}

The aphasia scores (MAST and BAT scores) did not differ between L1 and L2 for the overall score (12 patients, L1 mean score $=69.9$, L2 mean score $=66.4$, Wilcoxon, $z<1)$, the comprehension (L1 mean score $=39.3$, L2 mean score $=36.6, p=0.07$, $z=-1.83$ ) and the production subscore (L1 mean score $=28.6$, L2 mean score $=28.9, z<1)$ (see Table $2 \&$ Fig. 1$)$. When L1 and L2 performances of individual patients were compared, 11 out of the 12 patients did not present any statistically significant differences in any of the tasks. Only one patient (No. 11) showed a lower score in L2 than in L1 in the total MAST scores $(p=.0003)$, the comprehension $(p=0.004)$ and the production subscores $(p=.0007)$. Concerning naming, no relevant difference was found between L1 and $\mathrm{L} 2$ performances (Wilcoxon $p=0.86, z=0.18$ ).

\subsection{Comparison of anterior and posterior lesions}

The site of the lesion had no influence on this pattern: the total MAST score did not differ between L1 and L2 in either anterior (Wilcoxon $z<1$ ) or posterior (Wilcoxon $z<1$ ) lesions.

\subsection{Syntactic impairment and other language modalities}

At group level, syntactic performance was better in the first than in the second language $(p=0.004 ; z=2.903)$. The mean score for L1 was 28.9 (out of a maximum score of 40 ) and 24.6 for L2. At an individual level, four out of the 12 patients showed significant inter-language dissociation at the syntactic judgment task, showing superior score in L1 (for details and statistics, see Fig. 1). Eight patients showed an equal performance in both languages.

\subsection{Anatomo-syntactic analysis}

Among the 12 patients, eight were categorized in the " $A$ " (anterior) subgroup, and eight in the " $P$ " (posterior) subgroup. Four patients with AP lesions were included in both groups. We analyzed performances in the syntactic tasks in each subgroup, and compared performances for L1 and L2. Then, in a single-case approach, we defined if a L1/L2 dissociation for syntax was present or not in each patient.

When the site of the lesion was taken into account, analysis showed that the " $A$ " sub-group had significantly higher perfor-

Table 1

Summary of data in 12 late bilingual patients.

\begin{tabular}{|c|c|c|c|c|c|c|c|c|c|c|}
\hline Case no. & Age (yrs) sex & Site of lesion ${ }^{a}$ & & $\mathrm{~L} 1^{\mathrm{c}}$ & $\mathrm{L} 2^{\mathrm{C}}$ & Proficiency L2 ${ }^{\mathrm{b}}$ & Immersion in L2 & $\mathrm{AOA}^{\mathrm{d}}$ & Aphasia type $\mathrm{e}^{\mathrm{e}}$ & Timing of assessment ${ }^{f}$ \\
\hline 1 & 69, M & FTI, capsular & A & Ita & Fre & High & Low & 23 & Broca & $5 \mathrm{w}$ \\
\hline 2 & $66, M$ & Post junctional & $\mathrm{P}$ & Ita & Fre & Low $(0.45)$ & Low & 25 & Mixed & 7 th d \\
\hline 3 & $39, \mathrm{~F}$ & Superficial F & A & Spa & Fre & High $(0.86)$ & High & 24 & Broca & 18th d \\
\hline 4 & $68, \mathrm{M}$ & TP, bilat & $\mathrm{P}$ & Ita & Fre & Low $(0.33)$ & Low & 24 & Transc mixed & $18-26 \mathrm{~d}$ \\
\hline 5 & $78, \mathrm{M}$ & F, opercular, insular & A & Ita & Fre & Low (0.52) & Low & 17 & Transc motor & 5 th d \\
\hline 6 & $63, \mathrm{M}$ & FP & $\mathrm{AP}$ & Ita & Fre & High (0.67) & Low & 18 & Mixed & 8th d \\
\hline 7 & $63, \mathrm{~F}$ & $\mathrm{~F}$ & $\mathrm{~A}$ & Ger & Fre & High (0.93) & Low & 19 & Conduction & $16-17 \mathrm{~d}$ \\
\hline 8 & $78, \mathrm{M}$ & FP & $\mathrm{AP}$ & Ger & Fre & High $(0.78)$ & Low & 12 & Global & $18 \mathrm{~m}$ \\
\hline 9 & $66, \mathrm{M}$ & Fronto-insulo-temporal & $\mathrm{AP}$ & Ita & Fre & Low $(0.47)$ & Low & 25 & Mixed & $2.5 \mathrm{~m}$ \\
\hline 10 & $70, \mathrm{M}$ & $\mathrm{TP}$, insula & $\mathrm{P}$ & Ita & Fre & High (0.67) & High & 22 & Wernicke & $20-21 \mathrm{~d}$ \\
\hline 11 & $81, \mathrm{~F}$ & Post, para-hippocampus & $\mathrm{P}$ & Ger & Fre & High (0.67) & Low & 10 & Transc sensory & $20-25 d$ \\
\hline 12 & $68, \mathrm{~F}$ & FP & $\mathrm{AP}$ & Ger & Fre & High (0.79) & Low & 12 & Global & 9 th d \\
\hline
\end{tabular}

${ }^{\mathrm{a}} \mathrm{A}=$ anterior, $\mathrm{AP}=$ antero-posterior, $\mathrm{P}=$ posterior, post $=$ posterior, $\mathrm{T}=$ temporal, $\mathrm{F}=$ frontal .

b L1 scored 1.0 by definition.

c Ita $=$ Italian, Fre $=$ French, Spa $=$ Spanish, Ger $=$ German, Eng $=$ English.

d $\mathrm{AOA}=$ age of acquisition of the second language.

e Transc $=$ transcortical.

${ }^{\mathrm{f}}$ After stroke onset, $\mathrm{d}=$ day, $\mathrm{w}=$ week, $\mathrm{m}=$ month, $\mathrm{y}=$ year.

Table 2

Language data (Percent correct).

\begin{tabular}{|c|c|c|c|c|c|c|c|c|c|c|c|c|}
\hline \multirow[t]{2}{*}{ Case no. } & \multicolumn{3}{|c|}{ Language performances (MAST/BAT total) } & \multicolumn{3}{|c|}{ Expression task } & \multicolumn{3}{|c|}{ Comprehension task } & \multicolumn{3}{|c|}{ Syntactic task } \\
\hline & L1 & L2 & $p \#$ & L1 & L2 & $p \#$ & L1 & L2 & $p \#$ & L1 & L2 & $p \#$ \\
\hline 1 & 78 & 76 & ns & 60 & 57 & $n s$ & 100 & 100 & $n s$ & 80 & $55^{\mathrm{a}}$ & .017 \\
\hline 2 & 86 & 79 & $n s$ & 80 & 78 & $n s$ & 92 & 80 & ns & 60 & 50 & ns \\
\hline 3 & 90 & 90 & $n s$ & 84 & 88 & ns & 96 & 92 & $n s$ & 90 & 75 & $n s$ \\
\hline 4 & 84 & 74 & .08 & 84 & 76 & $n s$ & 84 & 72 & $n s$ & 60 & 63 & $n s$ \\
\hline 5 & 83 & 75 & $n s$ & 78 & 66 & ns & 88 & 84 & $n s$ & 88 & $58^{\mathrm{a}}$ & .003 \\
\hline 6 & 59 & 67 & $n s$ & 50 & 66 & $n s$ & 68 & 68 & $n s$ & 53 & 50 & $n s$ \\
\hline 7 & 58 & 59 & $n s$ & 20 & 26 & $n s$ & 96 & 92 & $n s$ & 88 & $65^{a}$ & .018 \\
\hline 8 & 83 & 82 & $n s$ & 74 & 76 & $n s$ & 92 & 88 & ns & 100 & $85^{\mathrm{a}}$ & .002 \\
\hline 9 & 43 & 47 & $n s$ & 34 & 42 & $n s$ & 52 & 52 & $n s$ & 53 & 50 & $n s$ \\
\hline 10 & 26 & 31 & $n s$ & 4 & 14 & $n s$ & 48 & 48 & ns & 45 & 55 & $n s$ \\
\hline 11 & 64 & $38^{\mathrm{a}}$ & .0003 & 60 & $30^{\mathrm{a}}$ & .0007 & 68 & $44^{\mathrm{a}}$ & .003 & 68 & 53 & $n s$ \\
\hline 12 & 85 & 79 & $n s$ & 74 & 62 & $n s$ & 96 & 96 & $n s$ & 85 & 80 & $n s$ \\
\hline
\end{tabular}

Note: $\# p$ is established through the chi-squared distribution.

${ }^{a}$ Values which are significantly worse than in the other language. 


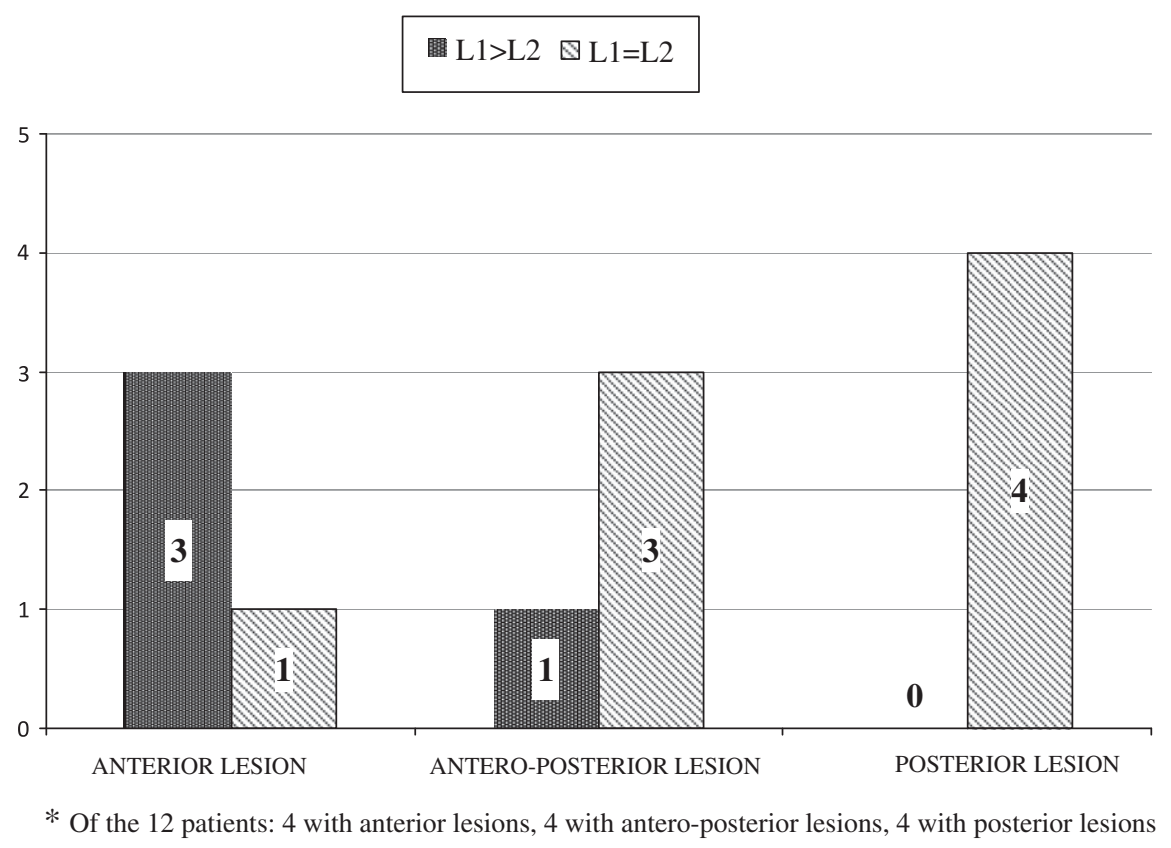

Figure 1. Relative syntactic performances in the 12 aphasic patients. In four patients with anterior involvement of the lesion the syntactic scores were higher in L1 than in L2, while this was not the case in patients with posterior lesions. No patient had scores L2 $\geqslant$ L1.

mance in L1 than in L2 (L1 syntactic mean score $=31.8$, L2 syntactic mean score $=25.9$, Wilcoxon, $p=0.018, z=-2.5$ ). No such differences were found in the "P" subgroup (L1 syntactic mean score $=26.1$, L2 syntactic mean score $=24.3, p=0.14, z=-1.5$ ).

Better performances in L1 were found in patients with anterior lesions only $(z=-2.5, p<0.02)$. Notably, a dissociation between L1 and L2 was found in three out of the four patients with a pure anterior lesion (Nos. 1, 5, 7), in the sense of better syntactic performance in L1. However, the same dissociation was found in only one of the four patients with AP lesions (No 8), and none of the patients with a pure P lesion (Fig. 1)

Analysis of the relation between the aphasia score, the performance in comprehension subscore and the site of the lesion revealed no difference between L1 and L2 and the localization of the lesion. The MAST mean score in the anterior subgroup was 67.1 in L1 and 66.9 in L2 (Wilcoxon, $p=0.92, z=0.104$ ), while for the posterior subgroup the MAST mean score was 66.3 in L1 and 62.1 in $\mathrm{L} 2(p=0.32, z=0.98)$.

\subsection{Other influence factors}

In order to investigate the role of age of acquisition on syntactic performances, we compared the syntactic performance of the "medium-late" AoA group (10-18 years old: five patients, Nos. $5,6,8,11,12)$ and of the "late-late" AoA group (19-25 years old: seven patients, Nos. 1,2,3,4,7,9,10). Results showed lower syntactic performances in L2 in the medium-late group $(z=-2.032$, $p=0.042)$ but not in the "late-late" group $(z=-1.527, p=0.127)$. In both groups, only patients with anterior lesions showed inferior performances in L2: patients Nos. 5(A) and 8 (AP) in the mediumlate and patients Nos. $1(\mathrm{~A})$ and $7(\mathrm{~A})$ in the late-late.

Regarding proficiency in the second language, we did not find any difference between the subjects with high (eight patients, =HP) or low proficiency (four patients, $=\mathrm{LP}$ ) in the MAST aphasia test (HP mean score $=65.3$, LP mean score $=68.8$, Wilcoxon $p=0.46$, $z=0.73$ ), the comprehension subtest (HP mean score $=36.9$, LP mean score $=36.0$, Wilcoxon $p=0.46, z=0.73$ ), the expression subtest $(\mathrm{HP}$ mean score $=25.3$, LP mean score $=32.8$, Wilcoxon $p=0.28$, $z=1.07$ ), and the oral syntactic task (HP mean score $=25.9$, LP mean score $=22.0$, Wilcoxon $p=0.27, z=1.09$ ). Moreover, two out of three pure anterior patients with impaired L2 syntax were highly proficient and three out of four pure $P$ patients (none of whom had a dissociation), were lowly proficient.

\section{Discussion}

The aim of this prospective pilot study was to compare the severity of aphasic symptoms in a specific bilingual stroke population, i.e., late L2 acquisition bilinguals. Our results may be summarized as follows: (i) no difference was found between L1 and L2 in the aphasia total score; (ii), however, greater L2 syntactical impairment was found, mostly in patients with anterior lesions, which contradicts previous proposals as to the role of posterior areas in late L2 syntactic processing (Ullman, 2001); (iii) better preservation of L1 syntactic processing was found only in patients with anterior lesions.

Globally seen, L1 and L2 were equally impaired in 11 of the 12 patients, and L2 was more impaired than L1 for only one subject. The lack of difference between L1 and L2 in global aphasia performance is compatible with the Parallel Impairment hypothesis (Green, 1995) and in line with previous group studies on bilingual aphasics (Fabbro, 2001; Paradis, 2001). Likewise, Vilarino (1997, 49 early bilinguals) and Roberts (1998, 16 aphasic bilinguals) did not find selective loss of either language. Only Junqué, Vendrell, and Vendrell's (1995, 50 aphasic bilinguals) data reported a trend towards a better preservation of L1, possibly due to higher exposure to L1 (Blonder et al., 2005).

Our results, which point to similar global impairment in L1 and L2, are also in line with current fMRI studies (see for review: Abutalebi, 2008; Indefrey, 2006) and clinical analysis (see Miozzo, Costa, Hernández, \& Rapp, 2010) suggesting that L1 and L2 are organized in a similar fashion at the brain level. Since we did not demonstrate any differences in expression, comprehension and syntactic performances between patients with low and high L2 proficiency, this pattern seems not to be influenced by the level of proficiency. Abutalebi (2008) suggested that L2 is acquired 
through the same neural structures responsible for L1 processing, independently of the linguistic domains (lexical-semantic, syntactic and phonologic processing). The rationale for this is that once a distinct brain area becomes specialized in a particular type of computation in L1 (such as left Broca's area and subcortical structures for syntactical processing), it will carry out the same type of computation if another language is added, even if that language is added later in life (Abutalebi \& Green, 2007). Abutalebi and Green (2007) admitted that, in certain cases, the learning of a second language may entail additional brain activity, especially in those cases where L2 is processed with a lower degree of proficiency. However, in these specific cases the additional L2 activity is mostly localized in prefrontal areas and related to the cognitive effort the L2 speaker needs to make to perform a task in L2. Taking the overlapping brain representation for L1 and L2 as granted, it may follow that a brain lesion should affect L1 and L2 to the same degree.

As for specific linguistic domains such as syntactical processing, it is interesting to observe the direct data comparisons of our individual patients: among the 12 patients, four had a significantly inferior score in L2 and only eight had equivalent scores. None had higher "syntactic" scores in L2. Such data may indicate that syntactical judgment in late bilingual patients may be more affected than other language tasks. With regard to syntactical processing, we also found that lesion sites may indeed play a consistent role in L2 syntactic impairment as originally postulated by Ullman (2001). However, our data point indicate otherwise, i.e., anterior rather than posterior lesion localizations are mostly associated with L2 syntactical impairments. Indeed, in all four patients with L2 syntactic impairments (of whom only one also had post-rolandic involvement), the infarctions were localized in pre-rolandic areas.

Thus we could not confirm the anterior/posterior dichotomy for L2 syntax processing in late bilinguals as postulated by Ullman (2001). The association between left anterior lesions - particularly Brocas's area, the left posterior frontal operculum and the left putamen - and syntactical impairments is well known in monolinguals (Friederici, Rüschemeyer, Hahne, \& Fiebach, 2003; January, Trueswell, \& Thompson-Schill, 2008; Metlzer, McArdle, Schafer, \& Braun, 2009). Interestingly, our data suggest that L1 and L2 syntactic processing seems to be dependent on comparable structures but that L1 is relatively better preserved following a lesion. One possibility for this unexpected finding is that, as hypothesized by Green and Abutalebi (2008), late bilingual speakers may rely on more cognitive control resources to process their L2 since, unlike L1, a later-learned L2 is not processed in an automatic manner. L2 syntactical processing is a more demanding and controlled process than L1, especially in the case of late L2 AoA. The cognitive control resources necessary for supporting L2 processing reside mainly in the frontal lobe and subcortical structures (Abutalebi \& Green, 2007), and therefore an anterior lesion may also have repercussions on these control processes (for recent functional connectivity evidence for this hypothesis in bilingual aphasia, see Abutalebi, Della Rosa, Tettamanti, Green, \& Cappa, 2009). The fact that we observed this clinical dissociation between L1 and L2 syntactical processing in our current patients may indicate precisely that L1 syntactical processing is more consolidated in the left frontal lobe, and hence less susceptible to brain damage. On the other hand, L2 syntax is more susceptible because it requires additional cognitive resources in the frontal lobe; resources that are no longer available due to the lesion. This interpretation is supported by fMRI data collected during bilingual syntactical production which shows additional left inferior frontal activation in L2 in subjects with late acquisition and with less proficient L2 (Golestani et al., 2005; Sakai, Miura, Narafu, \& Muraishi, 2004; Weber \& Indefrey, 2009).
In conclusion, this pilot study suggests that syntactical impairments in bilingual aphasia may specifically affect L2, especially if L2 is acquired after the critical period. Crucially, the lesion site may influence this clinical picture, in the sense that it is most probable that anterior lesions will produce L2 related syntactical deficits.

\section{Methods}

\subsection{Subject population}

Twelve bilingual participants with stroke-induced aphasia were recruited for the study. All of them had French as L2 while L1 was Italian (seven patients), German (four) or Spanish (one). All the subjects [four females, eight males; age range 39-81 years (mean of 67.4, $\mathrm{SD}=10.7)$ ] were right-handed, except for one who was ambidextrous. Inclusion criteria were: late acquisition ( $>6$ years) of L2, single left hemisphere stroke, sufficient proficiency of L2 for everyday conversation (B1 level of the Council of Europe), lesion demonstrated by neuroimaging and availability of information regarding prior language proficiency. Exclusion criteria were: left-handedness, prior cognitive impairment or focal brain lesion and inability to understand the tasks. Detailed patients characteristics with aphasia subtypes are provided in Table 1. All patients gave written, informed consent and the study was approved by the local Ethics Committee.

\subsection{Materials}

Premorbid biographical and linguistic factors: AoA was recorded directly from each subject and confirmed by the family. Premorbid proficiency and immersion in L2 were assessed by means of a questionnaire (Wartenburger et al., 2003) submitted to the patients and to their families (see Appendix 1 for the questionnaire and a summary of the results). A visual analogue scale was used to evaluate the proficiency of L2 (in speaking, reading, oral understanding and writing), and was then transcribed in relative values. The assessment of exposure to L2 included the following items: use of language at work, with friends, with family, watching TV or listening to the radio. We separated the $\mathrm{L} 2$ immersion values into two groups: high $(>50 \%)$ and low $(<50 \%)$ immersion.

Aphasia evaluation: Aphasia diagnosis was assessed in French through standard clinical evaluations (BDAE/Boston Diagnosis Aphasia Evaluation and MT Montreal Toulouse, see Goodglass, Kaplan, \& Barresi, 2001; Nespoulous et al., 1992). Aphasia severity was assessed using the same clinical evaluation in L1 and in L2. Eleven subjects were assessed using the Mississippi Aphasia Screening Test (MAST, total score of 100, Nakase-Thompson et al., 2005), and one patient (No. 1), for a practical reason, was evaluated using a shorter version of the Bilingual Aphasia Test (BAT, total score of 68, Paradis, 1987). The MAST test included nine tasks which assessed oral/written expression and comprehension (see Appendix 2; see also http://www.tbims.org/combi/mast/index.html). The MAST test was translated into four languages and internally validated through reversed translation by an external translator (see Appendix 2 for details). For the BAT test, we used the certified translated versions. Raw scores were used.

Syntactic judgment task: We created an auditory morpho-syntactic judgment task with 40 simple active sentences in each language, which were adapted from Hahne and Friederici (2002). Sentences were constructed using medium-to-high frequency words and focused on subject-verb or adjective-noun agreement; half of them were grammatically correct and the other half contained a morpho-syntactic violation (for example: "The cow are in the field"; see Appendix 3 for detailed description and sentences used). Only morpho-syntactic agreement/disagreement conditions resulting in clear phonological differences were included. For in- 
stance, French singular/plural contrast le-les (lə] - [le]) were not included as some L2 speakers did not perceive this contrast. The 40 sentences were recorded by a native speaker of each language.

\subsection{Anatomical data}

Brain MRI or CT imaging was performed at the acute post-stroke stage for each patient and analyzed (without disclosing the patients names) by two of the authors (MT and JMA). Anterior lesions (if anterior to the rolandic sulcus) received the abbreviation "A", posterior lesions "P" (if posterior to the rolandic sulcus) and anterio-posterior lesions "AP". Using this type of analysis, a patient with AP lesion was represented in both subgroups.

\subsection{Experimental procedure}

Testing was carried out in two separate sessions, one in French and one in the other language (Italian, German, English or Spanish). Session order was pseudo-randomly alternated among patients. In each session the patient underwent the aphasia evaluation (MAST or BAT) plus the auditory syntactic task. The 40 sentences of the syntactic task were verbally presented using a computer and headphones. Each trial started with a warning message, asking the subject to push a button to launch the next item. The sentence was then presented and the participant had to choose whether the sentence was correct or not by pushing a "correct" or "wrong" button. 3 The whole evaluation (aphasia and syntax evaluation) lasted 3040 min in each language. The Biographical Factors questionnaire was completed by a relative. All participants were tested after the fifth day post-stroke; for the patients tested during the acute after-stroke stage (the first 3 weeks), we made sure that the time period between L1 and L2 evaluations was reasonably short (less ; than $24 \mathrm{~h}$ ) to avoid misinterpretations related to frequent rapid recovery during the first week.

\subsection{Statistical analysis}

Due to the small sample and the group distribution, we used non-parametric tests for the statistical analysis. At the group level, we compared the subjects performances in the syntactic judgment task and the total aphasia scores across languages with a Wilcoxon paired comparison test, and the anatomoclinical analyses with a Mann-Whitney independent comparison test. At the individual level, L1 and L2 total scores and syntactic scores were compared using a Pearson chi-square test with an alpha of $p<0.05$.

\section{Acknowledgments}

This study was supported by the SNF 325100-118362 to J.-M.A. The authors are grateful to M. Michel and N. Denereaz for helping with the evaluations (M.M. and N.D.) and construction of the task (N.D.).

\section{Appendix A. Supplementary data}

Supplementary data associated with this article can be found, in the online version

\section{References}

Abutalebi, J. (2008). Neural aspects of second language representation and language control. Acta psychological, 128(3), 466-478.
Abutalebi, J., Della Rosa, P. A., Tettamanti, M., Green, D. W., \& Cappa, S. F. (2009) Bilingual aphasia and language control: A follow-up fMRI and intrinsic connectivity study. Brain and Language, 109, 141-156.

Abutalebi, J., \& Green, D. (2007). Bilingual language production: The neurocognition of language representation and control. Journal of Neurolinguistics, 20, 242-275.

Ansaldo, A. I., Marcotte, K., Scherer, L., \& Raboyeau, G. (2008). Language therapy and bilingual aphasia: Clinical implications of psycholinguistic and neuroimaging research. Journal of Neurolinguistics, 21, 539-557.

Blonder, L. X., Heilman, K. M., Ketterson, T., Rosenbek, J., Raymer, A., Crosson, B., et al. (2005). Affective facial and lexical expression in aprosodic versus aphasic stroke patients. Journal of the International Neuropsychological Society, 11(6), $677-685$.

Fabbro, F. (2001). The bilingual brain: Bilingual aphasia. Brain and Language, 79, 210-211.

Friederici, A. D., Rüschemeyer, S. A., Hahne, A., \& Fiebach, C. J. (2003). The role of left inferior frontal and superior temporal cortex in sentence comprehension: Localizing syntactic and semantic processes. Cerebral Cortex, 13(2), 170-177.

Golestani, N., Alario, F. X., Meriaux, S., Le Bihan, D., Dehaene, S., \& Pallier, C. (2005). Syntax production in bilinguals. Neuropsychologia, 44, 1029-1040.

Goodglass, H., Kaplan, E., \& Barresi, B. (2001). Boston diagnostic aphasia examination. Stimulus Cards (3rd ed., ). Lippincott.

Green, D. W. (1995)Michel Paradis (Ed.). Aspects of bilingual aphasia. Brain and Language, 59(3), 531-535.

Green, D. W., \& Abutalebi, J. (2008). Understanding the link between bilingual aphasia and language control. Journal of Neurolinguistics, 21, 558-576.

Grosjean, F. (1992). Another view of bilingualisms. In R. Harris (Ed.), Cognitive processing in bilinguals (Vol. 13, pp. 51-62). Amsterdam.

Hahne, A., \& Friederici, A. D. (2002). Differential task effects on semantic and syntactic processes as revealed by ERPs. Cognitive Brain Research, 13, 339-356.

Indefrey, P. (2006). A meta-analysis of hemodynamic studies on first and second language processing: Which suggested differences can we trust and what do they mean? Language learning, 56, 279-304.

January, D., Trueswell, J. C., \& Thompson-Schill, S. L. (2008). Co-localization of stroop and syntactic ambiguity resolution in Broca's area: Implications to the neural basis of sentence processing. Journal of Cognitive Neuroscience, 21(12), 2434-2444.

Junqué, C., Vendrell, J., \& Vendrell, P. (1995). Differential impairments and specific phenomena in 50 Catalan-Spanish bilingual aphasic patients. In M. Paradis (Ed.), Aspects of Bilingual Aphasia (pp. 177-209). Pergamon Press.

Metlzer, J. A., McArdle, J. J., Schafer, R. J., \& Braun, A. R. (2009). Neural aspects of sentence comprehension: Syntactic complexity, reversibility, and reanalysis. Cerebral cortex, 10, 1093.

Miozzo, M., Costa, A., Hernández, M., \& Rapp, B. (2010). Lexical processing in the bilingual brain: Evidence from grammatical/morphological deficits. Aphasiology, 24, 262-287.

Nakase-Thompson, R., Manning, E., Sherer, M., Yablon, S. A., Gontkovsky, S. L., \& Vickery, C. (2005). Brief assessment of severe language impairments: Initial validation of the Mississippi aphasia screening test. Brain Injury, 19(9), 685-691.

Nespoulous, J. L., Lecours, A. R., Lafond, D., Lemay, A., Puel, M., Joannette, Y., et al. (1992). Protocole Montréal-Toulouse d'examen linguistique de l'aphasie (MT86). Isbergues, France.: L'Ortho-Edition.

Paradis, M. (2001). Bilingual and polyglot aphasia. Language and aphasia (pp 69-91). Amsterdam: Elsevier science.

Perani, D., \& Abutalebi, J. (2005). The neural basis of first and second language processing. Current Opinion in Neurobiology, 15, 202-206.

Roberts, P. M., \& Le Dorze, G. (1998). Bilingual aphasia: Semantic organization, strategy use, and productivity in semantic verbal fluency. Brain and Language, 65(2), 287-312.

Sakai, K. L., Miura, K., Narafu, N., \& Muraishi, Y. (2004). Correlated functional changes of the prefrontal cortex in twins induced by classroom education of second language. Cerebral Cortex, 14, 1233-1239.

Singleton, D. (2007). The critical period hypothesis: Some problems. Interlinguistica, $17,48-56$.

Ullman, M. T. (2001). The neural basis of lexicon and grammar in first and second language: The declarative/procedural model. Bilingualism and Cognition, 4, $105-122$.

Vilariño, I., Prieto, J. M., Robles, A., Lema, M., \& Noya, M. (1997). A study of bilingual Galician-Castillian aphasic patients [Article in Spanish]. Revista Neurologista, 25(144), 1165-1167.

Wartenburger, I., Heekeren, H. R., Abutalebi, J., Cappa, S. F., Villringer, A., \& Perani, D. (2003). Early setting of grammatical processing in the bilingual brain. Neuron, 37, 159-170.

Weber, K., \& Indefrey, P. (2009). Syntactic priming in German-English bilinguals during sentence comprehension. Neuroimage, 46(4), 1164-1172.

Weber-Fox, C. M., \& Neville, H. J. (1996). Maturational constraints on functional specialization for language processing: ERP and behavioral evidence in bilingual speakers. Journal of Cognitive Neuroscience, 8, 231-256.

Weekes, B. S. (2010). Issues in bilingual aphasia: An introduction. Aphasiology, 24, 123-125.

Zanini, S., Tavano, A., Vorano, L., Schiavo, F., Gigli, G. L., Aglioti, S. M., et al. (2004) Greater syntactic impairments in native language in bilingual Parkinsonian patients. Journal of Neurology, Neurosurgery, and Psychiatry, 75, 1678-1681. 